\title{
Active region oscillations
}

\author{
E. O'Shea ${ }^{1}$, D. Banerjee ${ }^{2}$, J. G. Doyle ${ }^{3}$, B. Fleck ${ }^{4}$, and F. Murtagh ${ }^{5}$ \\ 1 ESA Space Science Department, ESTEC Solar System Division, Keplerlaan 1, 2201 AZ, Noordwijk, \\ The Netherlands \\ 2 Centre for Plasma Astrophysics, K.U. Leuven, Celestijnenlaan 200 B, 3001, Heverlee, Belgium \\ 3 Armagh Observatory, College Hill, Armagh BT61 9DG, N. Ireland \\ 4 ESA Space Science Department, NASA/GSFC, Mailcode 682.3, Greenbelt, MD 20771, USA \\ 5 School of Computer Science, The Queen's University of Belfast, Belfast BT7 1NN, N. Ireland \\ e-mail: dipu@wis.kuleuven.ac.be, jgd@star.arm.ac.uk, bfleck@esa.nascom. \\ nasa.gov, f.murtagh@qub.ac.uk
}

Received 21 February 2000 / Accepted 4 January 2001

\begin{abstract}
We report here on an investigation of high frequency oscillations in active regions, carried out using high cadence observations of O v $629 \AA$, Mg IX $368 \AA$ and Fe XVI $335 \AA$ with the Coronal Diagnostic Spectrometer (CDS) on SOHO. Using the techniques of wavelet analysis on various temporal series datasets, we find that certain oscillation frequencies are favoured for each line. We find furthermore that a $\sim 5$ min oscillation signature is commonly present in all lines, suggesting a coupling of the photospheric driver with the transition region and coronal loop modes. We report on the tendency for higher frequency oscillations to be present at lower intensity values, suggesting that higher frequency oscillations occur in interloop regions or at loop boundaries, possibly as a result of some resonant absorption process. In addition, we find that the coronal lines of Fe XVI and Mg IX show more significant oscillations in the velocity than in the intensity, which suggests that in the velocity we measure additional non-compressive wave modes not visible in the intensity. As this effect is not seen in the transition region line of $\mathrm{OV}$ it would seem that these additional non-compressive modes are produced in and limited to the corona. We suggest that there are two main mechanisms responsible for the observed oscillations; either resonant Alfvén and/or fast kink waves or propagating slow magnetoacoustic waves, both present in coronal loops.
\end{abstract}

Key words. Sun: UV radiation: Sun: oscillations: Sun: waves: Sun: corona

\section{Introduction}

It is known from observations that the solar atmosphere is threaded with strong magnetic fields in the form of flux tubes, which form coronal loops, the transverse dimensions of which are much shorter than the longitudinal ones. The study of oscillations from these loops can provide clues to the understanding of coronal heating. In the literature, different theoretical mechanisms have been suggested as possible candidates for coronal heating (see reviews by e.g. Hollweg 1990; Narain \& Ulmschneider 1996). Observations of coronal oscillations can provide a useful diagnostic tool to test the validity of these theoretical predictions. The oscillations are observed as either the intensity oscillations of a spectral line or of the continuum or as the velocity oscillations in the Doppler profiles, each providing separate but distinct clues to the heating from different parts of the corona. In this paper we will concen-

Send offprint requests to: E. O'Shea, e-mail: eoshea@so.estec.esa.nl trate on those oscillations found in coronal active region datasets.

A large number of attempts have been made to detect oscillations in the corona. However, despite the existence of a large body of literature, the results are not conclusive. Koutchmy et al. (1983) reported Doppler velocity oscillations with periods near 300,80 and $43 \mathrm{~s}$, but found no prominent intensity oscillations from the measurement of the green coronal line at $5303 \AA$. McKenzie \& Mullan (1997) reported loop oscillations in YОНкон/SXT images, revealing marginal oscillations in brightness with amplitudes of $\sim 1 \%$ and periods of $10-62$ s. Ireland et al. (1999) presented an analysis of active region oscillations for several transition region and coronal lines as observed by CDS on soHo. Cowsik et al. (1999) reported on high frequency, low-amplitude continuum intensity oscillations in the solar corona during the total solar eclipse of 26 February 1998. Recently, Aschwanden et al. (1999) detected spatial oscillations of coronal loops with TRACE. These loops having periods ranging from 258 to $320 \mathrm{~s}$ were interpreted as 
Table 1. Details of the temporal series observations

\begin{tabular}{lllll}
\hline Dates & Datasets & $\begin{array}{l}\text { Pointing } \\
X, Y\end{array}$ & $\begin{array}{l}\text { Start } \\
\text { UT }\end{array}$ & $\begin{array}{l}\text { End } \\
\text { UT }\end{array}$ \\
\hline 24 July 1997 & s8486r00 & 279,358 & $19: 17$ & $19: 51$ \\
& s8486r01 & 301,358 & $19: 51$ & $20: 25$ \\
& s8486r02 & 249,357 & $20: 25$ & $20: 59$ \\
25 July 1997 & s8490r00 & 439,371 & $11: 30$ & $12: 04$ \\
& s8490r02 & 387,377 & $12: 37$ & $13: 11$ \\
& s8490r03 & 367,375 & $13: 11$ & $13: 45$ \\
13 April 1998 & s10850r03 & $-311,353$ & $07: 53$ & $08: 15$ \\
& s10850r04 & $-311,353$ & $08: 15$ & $08: 37$ \\
& s10850r05 & $-311,353$ & $08: 37$ & $09: 00$ \\
15 July 1999 & s16831r00 & 438,296 & $13: 03$ & $13: 37$ \\
& s16831r01 & 438,296 & $13: 37$ & $14: 10$ \\
15 July 1999 & s16833r00 & 488,208 & $14: 25$ & $14: 58$ \\
& s16833r01 & 488,208 & $14: 58$ & $15: 32$ \\
16 July 1999 & s16844r00 & 661,320 & $21: 15$ & $21: 48$ \\
& s16844r01 & 661,320 & $21: 48$ & $22: 22$ \\
16 July 1999 & s16846r00 & 711,225 & $22: 36$ & $23: 10$ \\
& s16846r01 & 711,225 & $23: 10$ & $23: 43$ \\
\hline
\end{tabular}

kink mode magnetohydrodynamic standing waves. They also compiled a very useful summary of previous temporal observations, methods and theoretical interpretations in a tabular format (see their Table 1). Using TRACE, Nakariakov et al. (1999) observed a decaying transversal oscillation of a long, thin, bright coronal loop in the Fe XI $171 \AA$ emission line. These oscillations were excited by a solar flare in an adjacent active region. The decay time of the oscillation was $14.5 \pm 2.7 \mathrm{~min}$ for an oscillation with a frequency of $3.9 \pm 0.13 \mathrm{mHz}$.

In this paper we present time series observations of OV $629 \AA, \operatorname{Mg}$ IX $368 \AA$ and FeXVI $335 \AA$ lines in active regions, as observed by the Coronal Diagnostic Spectrometer (CDS) onboard soHO. With these observations and the methods of wavelet analysis we examine intensity and velocity oscillations for evidence of different wave modes. The results of our analysis are then discussed and compared to different theoretical models available in the literature.

\section{Observations and data reduction}

To obtain these observations we used the Coronal Diagnostic Spectrometer (CDS) onboard the SOHO satellite and, in particular, the Normal Incidence Spectrometer (NIS). The data discussed here were obtained from three observing periods in 1997, 1998 and 1999. Further details of these observations are summarised in Table 1 . The 1997 and 1999 data were obtained using the observing sequence CHROM_N4, with a slit size of $4 \times 119 \operatorname{arcsec}^{2}$ and a cadence of $\sim 10 \mathrm{~s}$, while the 1998 data was obtained using the CHROM_HI observing sequence, with a slit size of $4 \times 89 \operatorname{arcsec}^{2}$ and a cadence of $\sim 7 \mathrm{~s}$. These sequences recorded data for three spectral lines, namely Ov $629 \AA$ $\left(\log T_{\mathrm{e}}=5.4 \mathrm{~K}\right), \mathrm{Mg} \mathrm{IX} 368 \AA\left(\log T_{\mathrm{e}}=6.0 \mathrm{~K}\right)$ and Fe XVI $335 \AA\left(\log T_{\mathrm{e}}=6.3 \mathrm{~K}\right)$. All datasets from each of the three years contained 200 time frames. The eight datasets from
15-16 July 1999 were obtained after the recovery of soHo and so the lines show the characteristic broadened wings. This broadening has the effect of blending the Mg IX $368 \AA$ line with that of the nearby Mg VII $367 \AA$ line. We were able to fit the $\mathrm{Mg}$ IX component of this blend satisfactorily by using a double Gaussian, and by fixing the position of the Mg VII line. In all other cases fitting was done using a single Gaussian as the lines were found to be generally symmetric. The fitting was done using the updated CFIT software available in the CDS software tree.

Rotational compensation was not used for any of the temporal series and the data was thus obtained in a sitand-stare mode. Due to the relatively large width of the slit (4 arcsec), and following the discussion of Doyle et al. (1998) for Fourier power spectra, we estimate that the effect of the sit-and-stare mode on the resulting (wavelet) power would be to spread the power over a frequency range of at most $\sim 1 \mathrm{mHz}$, depending on the size of the source and location of the active region.

Using the standard CDS software procedure VDS_CALIB we de-biased and flat fielded the data. The resulting data after running this procedure were in units of photon-events/pixel/sec. Multiplying by the exposure time yielded units of photon-events/pixel. The data was cleaned of cosmic ray hits by using the CDS software procedure CDS_CLEAN. Slant and tilt corrections were applied to the data using another CDS software procedure, NIS_ROTATE. To improve the signal-to-noise, the data was binned by three pixels along the slit, in effect creating new pixels of $\sim 5 \times 4 \operatorname{arcsec}^{2}$.

We subsequently obtained the total number of photonevents in a line by integrating under the fitted line profile. Note that in Sect. 3 these photon-events are referred to as "counts". The velocity values presented in this paper are relative velocities, that is, they are calculated relative to an averaged profile summed over all pixels along the slit and all time frames. This averaged profile was fitted by a Gaussian and its "rest" wavelength determined. All other profiles were then measured relative to this "rest" wavelength. No absolute calibration exists for the velocity scale, so that the derived velocities are therefore merely indicative of the presence of line shifts.

The localised (in time) nature of wavelet transforms allows the study of the duration of any statistically significant oscillations as well as their frequency/period. We use the software provided by Torrence \& Compo (1998), and we refer the reader to this paper for further details on the techniques of wavelet analysis involved. This wavelet software utilises the Morlet wavelet. It should be noted that the wavelet transform suffers from edge effects at both ends of the time series. The region in which these effects are important is defined by the "cone of influence" (COI). We use the definition of COI given by Torrence \& Compo (1998) but see also Meyers et al. (1993) for an alternative discussion.

To remove high frequency noise present in the data wavelet-based noise filtering was used. The CDS data used contains Poisson (detector) and Gaussian (fitting) noise 
(a)
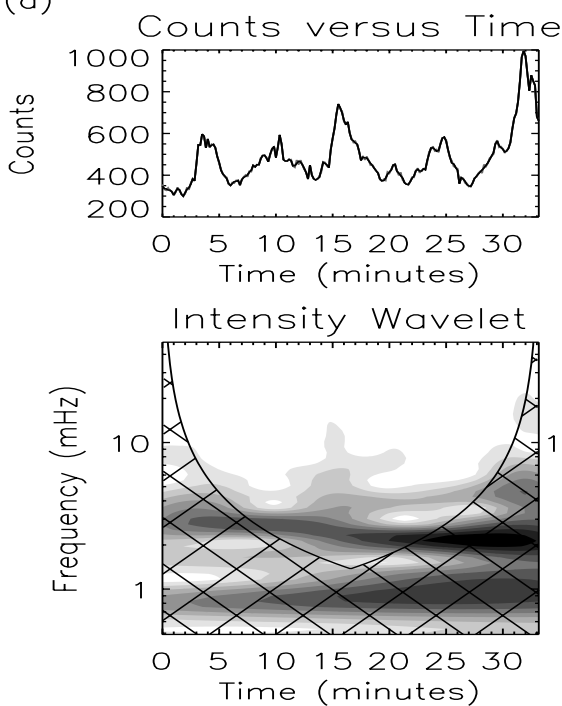

(b)
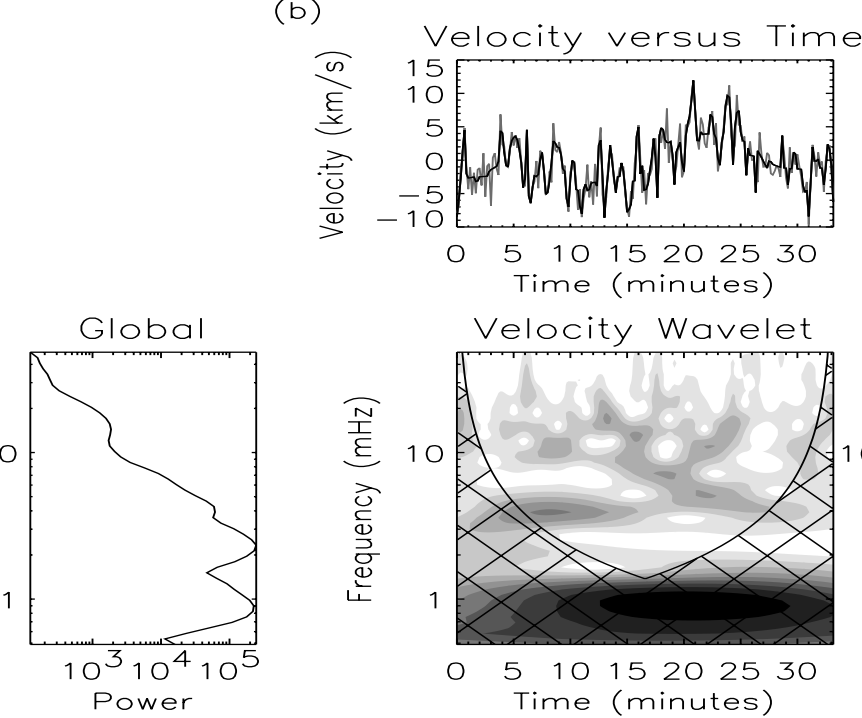

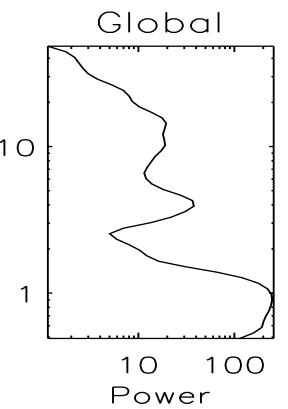

Fig. 1. a) The intensity time series, the corresponding wavelet power spectrum and its global wavelet spectrum for the Ov $629 \AA$ line at pixel position 3 in dataset s8486r02. b) The corresponding plots for the O v $629 \AA$ velocity values

(a)
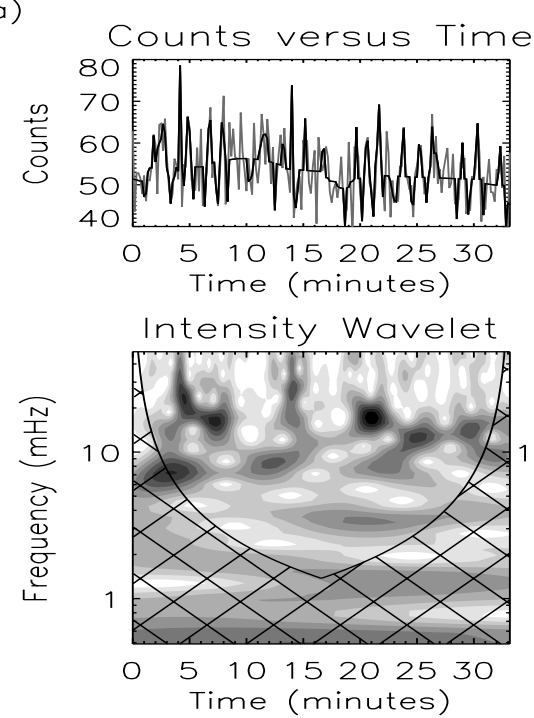

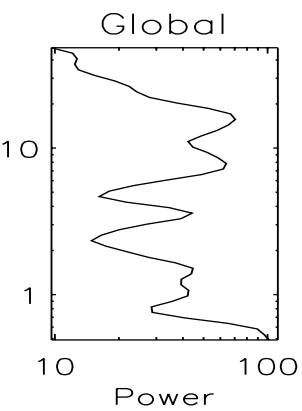

(b)
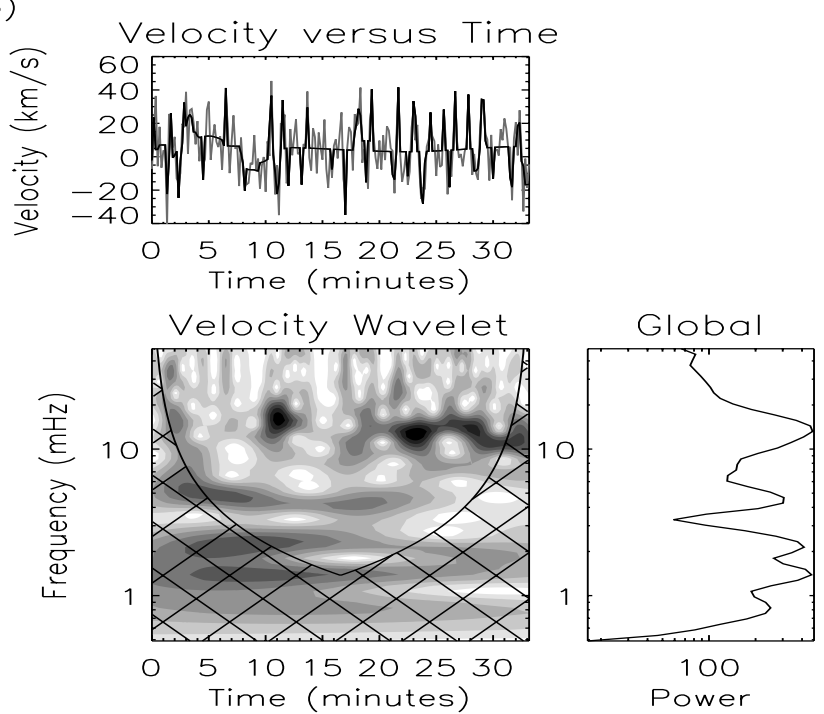

Fig. 2. a) The intensity time series, the corresponding wavelet power spectrum and its global wavelet spectrum for the MgIX $368 \AA$ line at pixel position 22 in dataset s8490r03. b) The corresponding plots for the Mg IX $368 \AA$ velocity values

components. This was modelled as Gaussian overall, on the grounds that (i) asymptotically Poisson noise becomes Gaussian, and (ii) the addition of Gaussians remains Gaussian. The principle of this is wavelet filtering is similar to filtering in Fourier space. First the signal is decomposed into wavelet space, the data is then filtered at each of the wavelet resolution levels (or scales), and then the signal is recomposed. This filtering was done using a noise estimation method similar to that discussed in Starck \& Murtagh (1998). That is, the standard deviation was estimated at each wavelet scale and hard thresholding was carried out in wavelet space, using a $2 \sigma$ detection $(\sim 95 \%)$ level. The particular wavelet transform used for this is a redundant transform known as the $B_{3}$ spline à trous wavelet transform. The wavelet mother function is rather like a Mexican hat, with a high central bump, negative side lobes, and is point symmetric. Further details and a background on many applications of this transform can be found in Starck et al. (1998). The associated MR/1 package was used in this work.

The statistical significance of the resulting filtered data was estimated by using a Monte Carlo or randomisation method. The advantage of using the randomisation method is that it is distribution free or non-parametric, i.e. it is not limited or constrained by any specific noise model, such as Poisson, Gaussian, etc. We follow the method of Fisher randomisation as outlined by Nemec \& Nemec (1985) and implemented in the UK Starlink software package, PERIOD (Dhillon \& Privett 1997). 
(a)
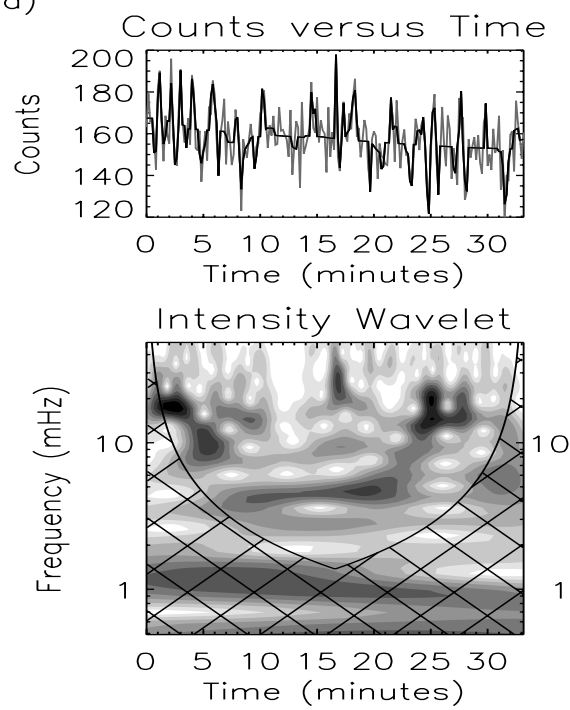

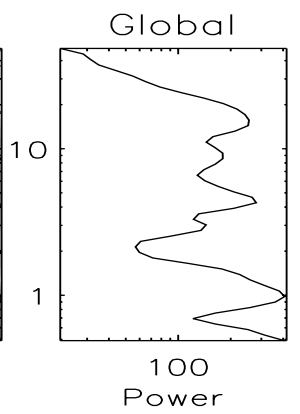

(b)
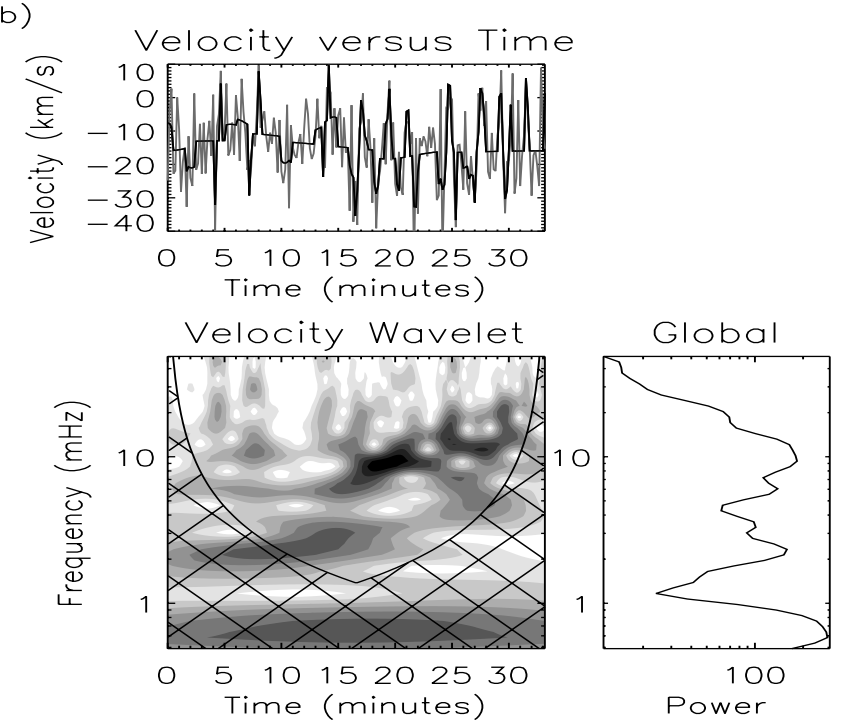

Fig. 3. a) The intensity time series, the corresponding wavelet power spectrum and its global wavelet spectrum for the FeXvI $335 \AA$ line at pixel position 11 in dataset s16844r00. b) The corresponding plots for the Fe XVI $335 \AA$ velocity values

We shall briefly discuss the method used in more detail below. The randomisation test is based on the assumption that, if there is no periodic signal in the time series data, then the measured values (intensity, velocity, etc.) are independent of their observation times. For example, the intensities $I_{1}, I_{2}, \ldots I_{n}$, observed at times $t_{1}, t_{2}, \ldots t_{n}$, are just as likely to have occurred in any other order $I_{r(1)}$, $I_{r(2)}, \ldots I_{r(n)}$, where $n$ is the total number of observations and $r(1), r(2), \ldots r(n)$ is a random permutation of the subscripts $1,2, \ldots n$. By using the maximum power peak in the global wavelet spectrum, which is just the average of the wavelet power over time and similar to a smoothed Fourier power spectrum (Torrence \& Compo 1998), at the "test statistic" (see Dhillon \& Privett 1997) it was possible to test the hypothesis that there was no periodicity in our data. Ideally this would have been done by evaluating the peak power from the original ordering of the time series data and then comparing it to the peak powers evaluated from the $n$ ! equally likely permutations of the time series data. The proportion of permutations that gave a value greater or equal to the peak power of the original time series would then provide an estimate of $p$, the probability that no periodic component is present in the data, i.e. a large value of $p$ suggests that there is little or no real periodicity in the data whereas a small value of $p$ suggests that the measured periodicity is likely to be real. In practise $n$ ! is usually so large that it is not possible to do this, due to computational and time constraints, and so the peak powers are generally calculated for only a random sample of $m$ permutations. By carrying out this approximation, for a random sample of 150 permutations, we were able to obtain a reliable estimate of $p$. For a sample of 150 random permutations the standard errors of the $p$ values are no greater than 0.04 (Nemec \& Nemec 1985). Of course, the larger the number of permutations chosen, $m$, the lower the standard error of the $p$ value.
The probability levels for this work are written as the values of $(1-p) \times 100$, i.e. the percentage probability that periodic components are present in the data. We arbitrarily choose a value of $95 \%$ as the lowest acceptable probability level. That is, only global wavelet spectra that show a power peak with a probability greater than $95 \%$ are deemed to be due to "real" oscillations in the time series sequences. Occasionally the estimated $p$ value can have a value of zero, i.e. there being an almost zero chance that the observed time series oscillations could have occurred by chance. In this case, and following Nemec \& Nemec (1985), the $95 \%$ confidence interval can be obtained using the binomial distribution, and is given by $0.0<p<$ 0.01 , that is, the probability $((1-p) \times 100)$ in this case is between $99-100 \%$.

\section{Results}

In this section we present the results of a wavelet analysis carried out on the different active region datasets. In Sect. 3.1, specific examples for selected regions are discussed while in Sect. 3.2 a detailed statistical analysis of all observed intensity and velocity oscillations is presented.

\subsection{Examples of wavelet analysis}

In Fig. 1 we plot the intensity and velocity time series, wavelet spectra and the global wavelet spectra for the $\mathrm{OV}$ line, at pixel position 3 in dataset s8486r02. In the time series of the intensity and velocity the (wavelet) filtered time series are shown as the bold line, while the unfiltered original is shown as the dotted grey line.

In the intensity time series in Fig. 1a we can see a strong 5-7 min period oscillating group. The frequencies corresponding to these oscillations are clearly shown in the wavelet spectrum plot underneath, where the darker 
colours indicate higher powers (the colour bar is inverted). Note that we do not consider frequencies lower than $2 \mathrm{mHz}$, as they are located in the COI and are therefore potentially affected by edge effects. From the wavelet spectrum plot we can see that the oscillation changes from being a $\sim 3.0 \mathrm{mHz}(\sim 5 \mathrm{~min})$ oscillation for the first $15 \mathrm{~min}$ to a longer $2.0-2.3 \mathrm{mHz}(\sim 7 \mathrm{~min})$ oscillation for the last $15 \mathrm{~min}$. In the global wavelet spectrum, which is the average of the wavelet spectrum over time (see Torrence \& Compo 1998), the main oscillation frequency, corresponding to the maximum power, is measured as $2.3 \mathrm{mHz}$, or a period of $7 \mathrm{~min}$. The second peak in the global wavelet spectrum, however, indicates that there is another weaker oscillation frequency present in this dataset at a frequency of $4.1 \mathrm{mHz}$ or $\sim 4 \mathrm{~min}$. Both these oscillations give probabilities of $99-100 \%$ using the randomisation method and therefore both of them are considered statistically significant (i.e. they are both $\geq 95 \%$ ). In Fig. 1b, the time series plot of the velocity clearly shows oscillations at a much higher frequency. This is confirmed in the wavelet spectrum where the dark contours indicate oscillations present between 3.0 and $20 \mathrm{mHz}$ ( $\sim 5$ min to 50 s periods). From the global wavelet spectrum at the right the main frequency of oscillation is measured from the maximum power peak to be $4.1 \mathrm{mHz}$, with a second weaker oscillation peak at $14.4 \mathrm{mHz}$. Note that in this case the randomisation test gives a probability of $95 \%$ for the $4.1 \mathrm{mHz}$ oscillation, but only $60 \%$ for the $14.4 \mathrm{mHz}$ oscillation. As such only the $4.1 \mathrm{mHz}$ oscillation is considered as being statistically significant here.

In Fig. 2 we carry out the same analysis for the Mg IX line at pixel position 22 in dataset s8490r03. Again in the plots of the intensity and velocity time series the filtered results are plotted as the bold line, while the unfiltered results are shown as a grey dotted line. In the intensity time series plot and below it in the wavelet spectrum plot it is possible to see that the main oscillations are occurring at frequencies of between 7.0 and $\sim 20 \mathrm{mHz}$. From the global wavelet spectrum the main frequency of oscillation for this line, measured from the peak power, is at $15.7 \mathrm{mHz}(\sim 1.1 \mathrm{~min})$, with a secondary oscillation at $7.5 \mathrm{mHz}(\sim 2.2 \mathrm{~min})$. These frequencies are considerably larger than those found in the $\mathrm{OV}$ line. However, from the randomisation test the $15.7 \mathrm{mHz}$ oscillation only has a probability of $81 \%$ and so is not considered statistically significant. The secondary oscillation of $7.5 \mathrm{mHz}$, on the other hand, has a probability of $96 \%$ and so is considered statistically significant.

If we look at the time series plot of the velocity, in Fig. 2b, we can see that it too has a high frequency of oscillation. The main oscillation frequency can be seen from the wavelet spectrum plot to be between 10 and $20 \mathrm{mHz}$. Note that these high frequency oscillations occur in short bursts lasting no more than $\sim 5 \mathrm{~min}$. From the global wavelet spectrum the main frequency of oscillation, measured from the main peak is at $13.8 \mathrm{mHz}(\sim 1.2 \mathrm{~min})$, with a probability of $97 \%$ from the randomisation test. The sec- ondary oscillation frequency is however much lower, at a value of $2.1 \mathrm{mHz}(\sim 8 \mathrm{~min})$, with a probability of $99-100 \%$.

In Fig. 3 we carry out a similar analysis for the Fe XVI line at pixel position 11 in dataset s16844r00. In the plot of the time series for the intensity shown in Fig. 3a, a rapidly oscillating group can be seen for the first 5 min. This is picked up in the wavelet spectrum plot as the dark contour at a little under $20 \mathrm{mHz}$. Another high frequency event is recorded at $25 \mathrm{~min}$, again lasting for only a few minutes. In the global wavelet plot the average of these frequencies turns out to be at $15.0 \mathrm{mHz}$, with a probability of $97 \%$. However, the main oscillation frequency in this time series, as measured by the global wavelet spectrum, is at a lower frequency of $4.5 \mathrm{mHz}$ (or a period of $\sim 3.7 \mathrm{~min}$ ). Interestingly this lower frequency oscillation occurs between the times of the higher frequency oscillations, i.e. between a time of 5 and $25 \mathrm{~min}$, as can be seen from the wavelet spectrum plot in Fig. 3a. However, this lower frequency of $4.5 \mathrm{mHz}$ is found not to be statistically significant having a probability of only $87 \%$.

In Fig. 3b we carry out the same analysis for the corresponding Fe XVI velocity. From the wavelet spectrum plot we can see that the velocity time series shows little high frequency oscillations until a time of $\sim 15 \mathrm{~min}$, when a strong oscillation at $\sim 9 \mathrm{mHz}$ begins, that then last for $\sim 5$ min. Slightly higher oscillations are then present for the rest of the observation time. The main frequency of oscillation is at $9.7 \mathrm{mHz}(\sim 1.7 \mathrm{~min})$, with a probability of $95 \%$, as measured from the strongest peak in the global wavelet spectrum, with the secondary frequency of oscillation at $2.2 \mathrm{mHz}(\sim 7.5 \mathrm{~min})$, with a probability of 99 $100 \%$.

It will be noticed from these few examples that the same frequencies are generally not present in the intensity and the velocity oscillations of each region. This is perhaps an indication of different wave modes being separately responsible for the intensity and velocity oscillations.

\subsection{Statistical analysis}

The data used in this analysis was obtained over three years and in three different active regions, at the coordinates shown in Table 1 . In total, 17 datasets were examined, 6 from 1997, 3 from 1998 and 8 from 1999. In this work we collect together the results from these three observing periods to provide a larger statistical sample. We do this in the hope of evaluating the most commonly occurring oscillations in active regions.

By using the global wavelet spectrum and the randomisation method (see Sect. 2) we investigated the main intensity and velocity oscillation frequencies at each slit position in each dataset. At each slit position the frequencies corresponding to the primary and the secondary peaks in the global wavelet spectrum were recorded, as was the value of the summed counts over time in the intensity time series being investigated. The primary and secondary oscillation frequencies were only accepted, as being due to 

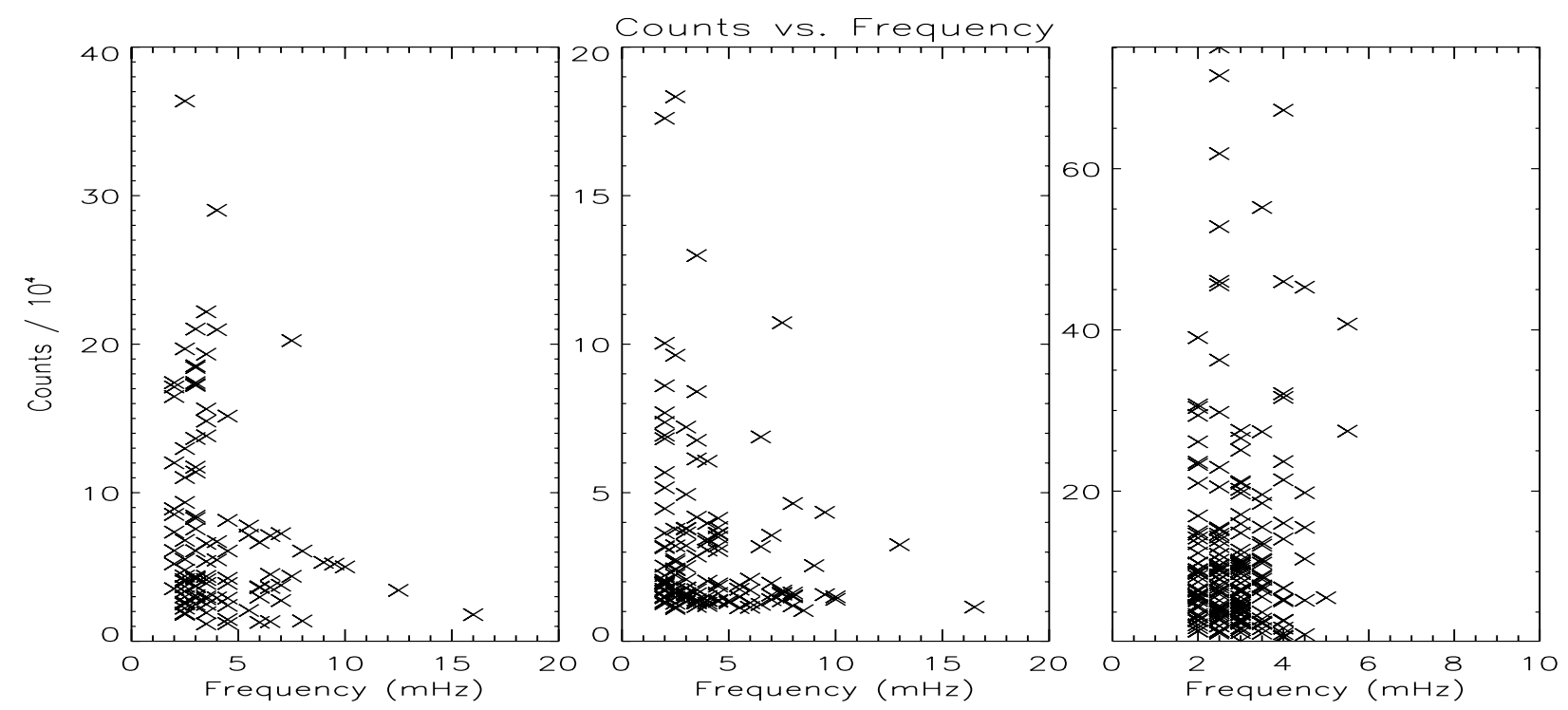

Fig. 4. The summed counts over time of the intensity time series versus the primary frequencies of oscillation found in these time series for Fe XVI $335 \AA$ (left panel), Mg IX $368 \AA$ (middle panel), O v $629 \AA$ (right panel). Note that the $x$-axis of the O V plot is only plotted up to $10 \mathrm{mHz}$
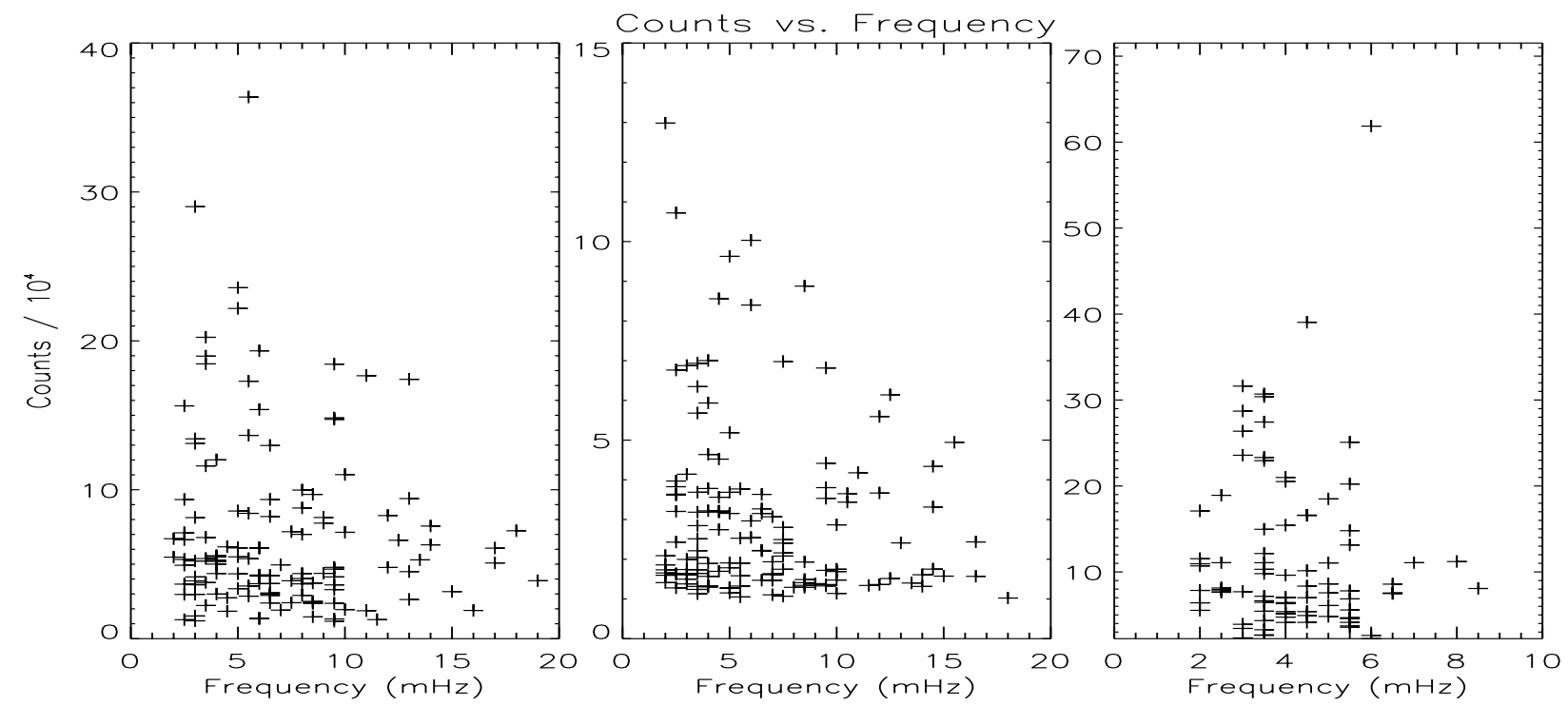

Fig. 5. The summed counts over time of the intensity time series versus the secondary frequencies of oscillation found in the these time series for Fe XVI $335 \AA$ (left panel), Mg IX $368 \AA$ (middle panel), O V $629 \AA$ (right panel). Note that the $x$-axis of the $\mathrm{O} v$ plot is only plotted up to $10 \mathrm{mHz}$

"real" oscillations, and therefore recorded, if the probability value from the randomisation method was greater than $95 \%$ in each case.

In this way we were able to build-up a list of all statistically significant intensity and velocity oscillation frequencies in the three lines for each dataset, together with an indication of the total intensity of the line where the oscillations were occurring. A frequency of $2.0 \mathrm{mHz}$ was chosen as the lower cut-off as frequencies below this value were judged to be in the COI and therefore potentially affected by edge effects. As the cadence in the 1997 and 1999 datasets is slightly different from that of the 1998 datasets, we rounded off all measured frequencies to the nearest $0.5 \mathrm{mHz}$, thus enabling the results for all datasets to be combined for a statistical study.

In Fig. 4 we plot the total intensity measured at each slit position versus the main frequency of oscillation in the intensity, measured from the primary peak in the global wavelet spectrum. Similarly, in Fig. 5 we plot the total intensity versus the secondary oscillation frequency found in the global wavelet spectra, i.e. frequencies corresponding to the second strongest peak in the global wavelet spectrum. (The oscillations measured from the primary and secondary peaks in the global wavelet spectra will subsequently only be referred to as the primary and secondary oscillations.) In Figs. 6 and 7 we plot the corresponding 

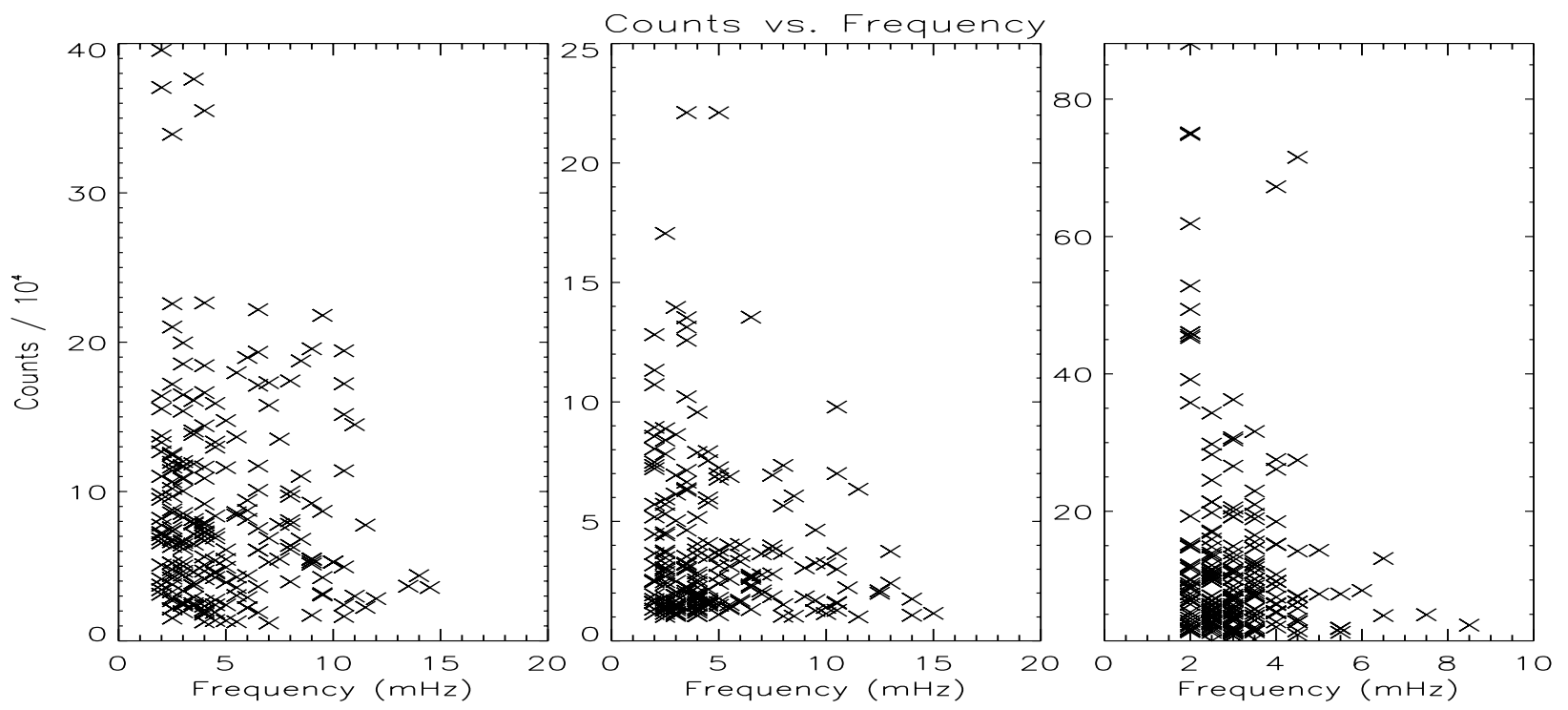

Fig. 6. The summed counts over time of the velocity time series versus the primary frequencies of oscillation found in these time series for Fe XVI $335 \AA$ (left panel), Mg IX $368 \AA$ (middle panel), O v $629 \AA$ (right panel). Note that the $x$-axis of the O V plot is only plotted up to $10 \mathrm{mHz}$
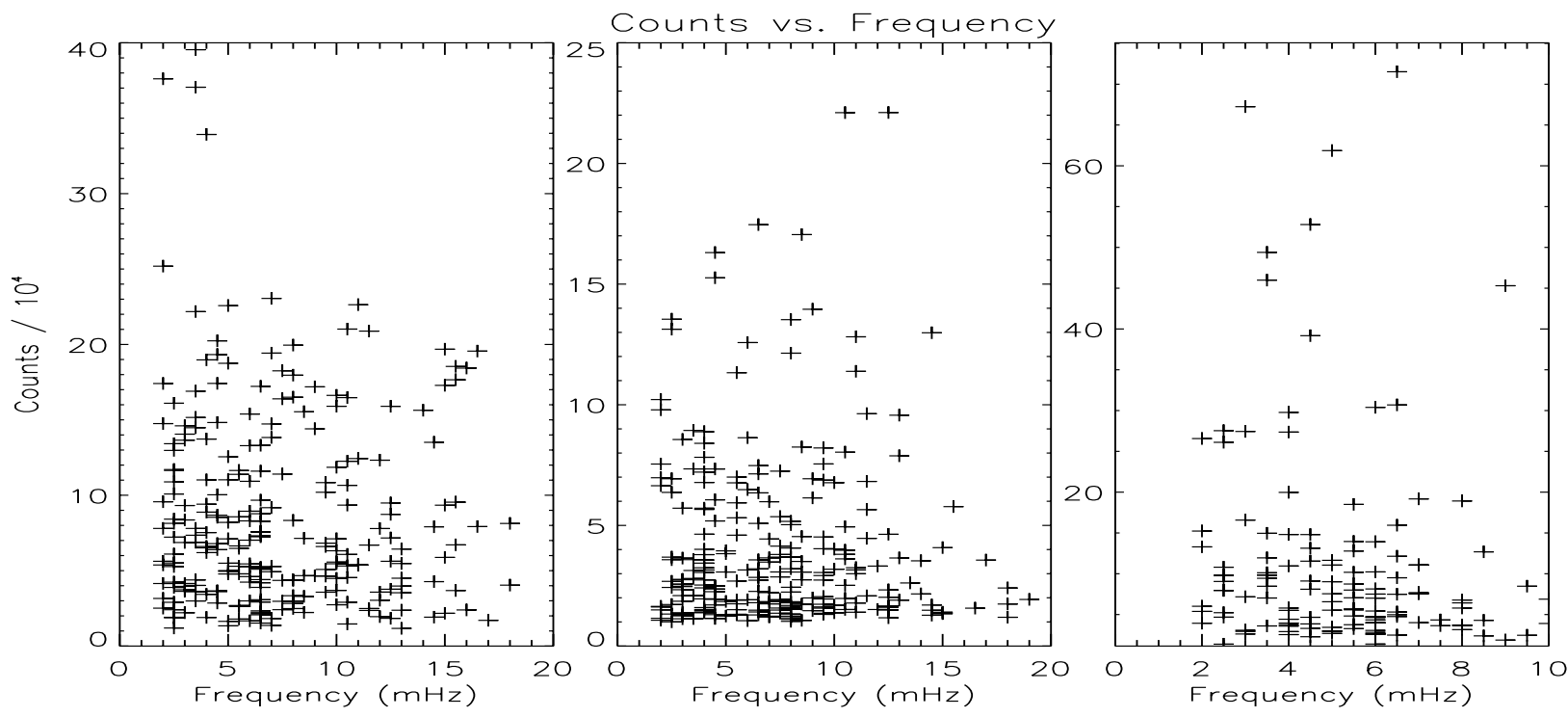

Fig. 7. The summed counts over time of the velocity time series versus the secondary frequencies of oscillation found in the these time series for Fe XVI $335 \AA$ (left panel), Mg IX $368 \AA$ (middle panel), O v $629 \AA$ (right panel). Note that the $x$-axis of the $\mathrm{O} v$ plot is only plotted up to $10 \mathrm{mHz}$

results for the velocity oscillations. By examining these plots it will be noticeable that the higher intensity values show a tendency to correspond to the lower frequency values. This is particularly clear by looking at the plots for the MgIX and Fe XVI lines in these figures. This suggests that the higher oscillation frequencies preferentially occur in the less bright parts of the active regions, such as the interloop regions.

Also noticeable is the fact that all lines show a slight change in the distribution of frequency in the primary and secondary oscillations. For example, while the Fe XVI and
Mg IX lines show similar distributions in both Figs. 4 and 5 , there are however slightly more of the secondary oscillations at higher frequencies and less at the lower frequencies. The distribution of the $\mathrm{OV}$ oscillation frequencies shows a bigger change. For example, in Fig. 5 the secondary oscillation frequencies of $\mathrm{OV}$ lie at values of between $3.0-6.0 \mathrm{mHz}$, in comparison with the primary oscillations plotted in Fig. 4 which lie at lower frequencies between $2.0-4.0 \mathrm{mHz}$. A similar effect is also seen in the frequencies measured from the velocity oscillations which are plotted in Figs. 6 and 7. Here again we can see that 
the distribution of $\mathrm{O} v$ frequencies changes and increases in value as we pass from the primary oscillations to the secondary oscillations.

To examine the overall distribution of frequencies in the intensity oscillations more carefully we plot, in Fig. 8, histograms of the frequencies obtained from the primary and secondary peaks in the global wavelet spectrum. The histograms of the primary oscillations are plotted in bold lines. From these primary oscillation histograms it can be seen that the most common frequencies found in each line lie between $2.0-5.0 \mathrm{mHz}$. In fact the histograms peak at $3.0 \mathrm{mHz}(\sim 5 \mathrm{~min})$ in the case of the Fe XVI and $\mathrm{O} v$ lines. Both the MgIX and Fe XVI lines also show separate peaks at $4.5 \mathrm{mHz}$. These results indicates that low frequency 2.0-5.0 $\mathrm{mHz}(\sim 3.3-8.3 \mathrm{~min})$ oscillations, typically centred at around $3.0 \mathrm{mHz}$ (or $\sim 5 \mathrm{~min}$ ) are commonly present in lines from the transition region as well as the corona. It may be that what we are seeing in these high temperature transition region and coronal lines is the influence of the photospheric five minute oscillations, which appear to be imprinting their characteristic periods of oscillation on to the upper atmospheric layers. From the histograms of primary oscillation frequencies it can be seen that the Mg IX and Fexvi lines also show some primary oscillations at higher frequencies between about 5.0 and $10.0 \mathrm{mHz}$. On the other hand, the transition region $\mathrm{O} v$ line shows very few, if any, oscillations at frequencies higher than $5 \mathrm{mHz}$.

If we now look at the histograms of the secondary oscillations (plotted as the thin lines in the same figure) we can see a different picture. There are now many more oscillations present at higher frequencies in each line. Examining the secondary oscillation histograms of the Fe XVI line we can see that there are now many more high frequency oscillations present, particularly in the range $5.0-10.0 \mathrm{mHz}$. The increase in the number of oscillations in the 8.0$10.0 \mathrm{mHz}$ range is particularly marked. Note, however, that there is still a sizeable peak in the histogram at $3.0 \mathrm{mHz}$. We see the same result in the $\mathrm{Mg}$ IX line where there is an increase in the number of oscillations with frequencies between $5.0-10.0 \mathrm{mHz}$. The results from the $\mathrm{O} \mathrm{V}$ line show the appearance of a new distribution of frequencies from $\sim 2.0-8.0 \mathrm{mHz}$, with peaks present in the histogram at 3.5 and $5.5 \mathrm{mHz}$. It is probable that the reason we do not see as many of these higher frequencies in the primary oscillations (e.g. Fig. 4) is that the strength of the primary oscillation generally has the effect of masking the presence of these weaker higher frequency oscillations. As the strength of the low frequency oscillations seem to have the effect of obscuring the weaker higher frequency oscillations, one way of seeing a more correct distribution of all measured oscillation frequencies would be to plot both the primary and secondary oscillations in a combined histogram plot.

We do this in Fig. 9. In this figure the histogram of the Fexvi line shows there to be a large number of frequencies between $2.0-5.0 \mathrm{mHz}$, peaking at $3.0 \mathrm{mHz}$. It also shows numerous higher frequencies present all the way up to $19.0 \mathrm{mHz}$. From the same figure it can be seen that the $\mathrm{Mg}$ IX line also has a large number of oscillations with frequencies in the $2.0-5.0 \mathrm{mHz}$ range, with the majority occurring at $2.0 \mathrm{mHz}$. Higher frequency oscillations are present up to $18 \mathrm{mHz}$, similar to the result found in the Fe XVI line. The histogram of the $\mathrm{O} v$ line shows that the majority of its oscillations occur at $3.0 \mathrm{mHz}$, together with a smaller number at $5.5 \mathrm{mHz}$, as evidenced by the peaks at these values. It is noticeable that the $\mathrm{OV}$ line does not show any oscillations with frequencies greater than $8.5 \mathrm{mHz}$.

In Fig. 10 we plot the histograms of the primary and secondary oscillations measured in the velocity. It can be seen for the primary oscillations of FexVI, plotted as the bold line histogram, that the most commonly measured frequency is at $2.5 \mathrm{mHz}$, with numerous $4.0 \mathrm{mHz}$ oscillations also being present. At higher frequencies the oscillations are mainly concentrated between $6.0-11.0 \mathrm{mHz}$ but there are frequencies present all the way up to $14.5 \mathrm{mHz}$. In the histogram for the secondary oscillations, plotted here as the thin line, there is still a peak at $2.5 \mathrm{mHz}$, but now also a stronger peak present at $6.5 \mathrm{mHz}$. In the case of these secondary oscillations it can be easily seen that there are now many more oscillations located at the higher frequencies, e.g. in addition to the strong $6.5 \mathrm{mHz}$ peak, there are now more oscillations present in the 10.0 $20.0 \mathrm{mHz}$ range.

The primary oscillation histogram of the Mg IX velocity measurements shows that the most commonly occurring frequency of oscillation is at $2.5 \mathrm{mHz}$. The strong peak in the histogram at $4.0 \mathrm{mHz}$ indicates that oscillations at this frequency are also common. At higher frequencies $(>5.0 \mathrm{mHz})$ there are oscillations present at all frequencies up to $15.0 \mathrm{mHz}$, with a slight increase in the number of oscillations at $\sim 10.0 / 10.5 \mathrm{mHz}$, where there is a small peak in the histogram. In the secondary oscillation histogram the main frequencies of oscillation are located typically at higher frequencies, with the largest number being present between $6.0-15.0 \mathrm{mHz}$. There are also numerous oscillations present with frequencies up to $20.0 \mathrm{mHz}$. Note however that there are also still a sizeable number of frequencies present at 3.0 and $4.0 \mathrm{mHz}$, as indicated by the peaks in the histograms.

In the $\mathrm{Ov}$ histogram it is clear that the most commonly occurring frequency is at $2.5 \mathrm{mHz}$, with a short tail of frequencies being present up to $8.5 \mathrm{mHz}$. In the secondary oscillations, on the other hand, there is a broad band of frequencies present from 2.0 to $16.5 \mathrm{mHz}$, with local peaks at 4.0 and $6.0 \mathrm{mHz}$. As in the intensity oscillations it is clear that the secondary oscillations for this line are present at higher frequencies with respect to the primary oscillations. Again this probably indicates that the strong primary oscillations of $\mathrm{OV}$ have the effect of blocking out these weaker higher frequency oscillations.

Note that we can again see in these velocity oscillations that the main frequencies of oscillation lie between 2.0 $5.0 \mathrm{mHz}$, for all the lines. Thus it appears that the velocity measurements are also being affected by some process 

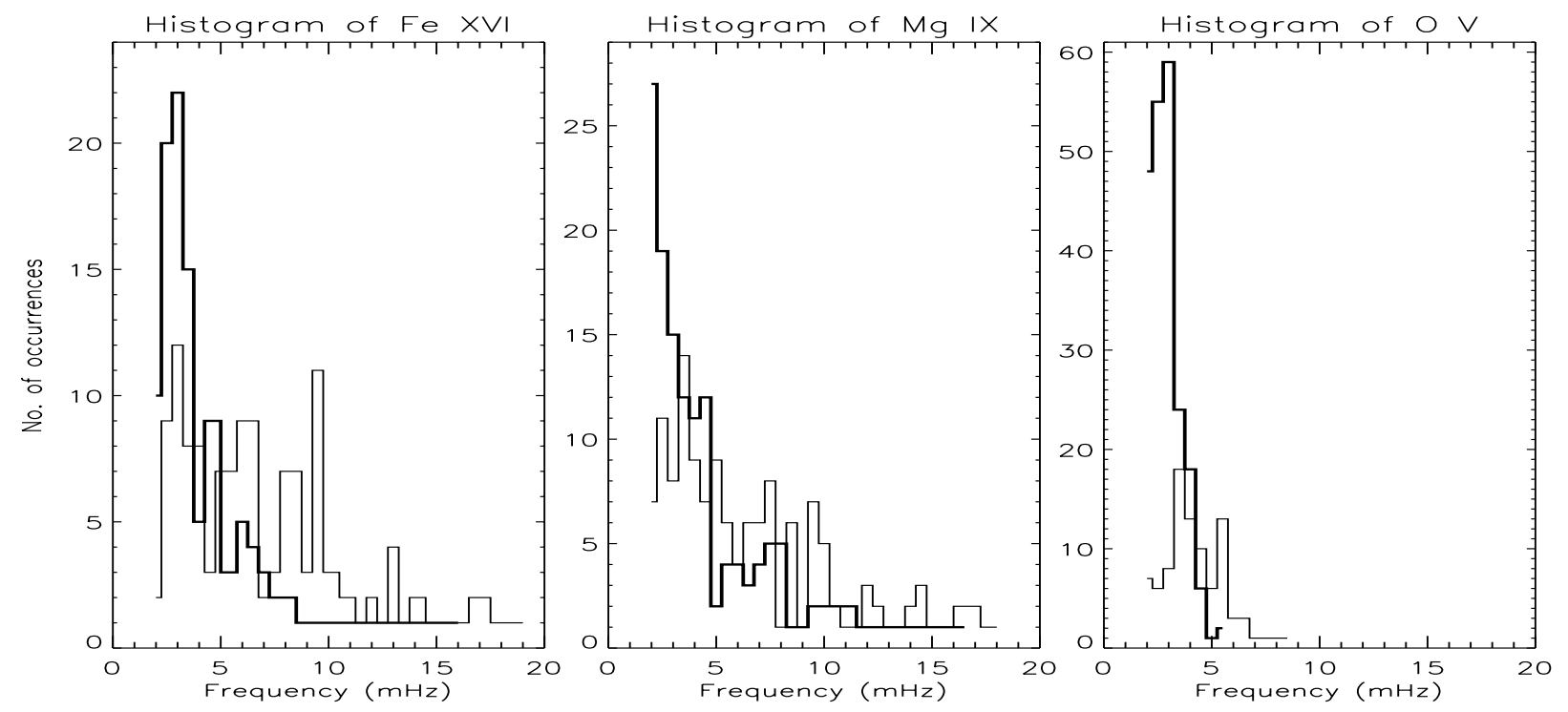

Fig. 8. Histograms of primary oscillation frequencies (bold line) and secondary oscillation frequencies (thin line), obtained from the intensity time series of Fe XVI $335 \AA$ (left panel), Mg IX $368 \AA$ (middle panel) and OV $629 \AA$ (right panel)
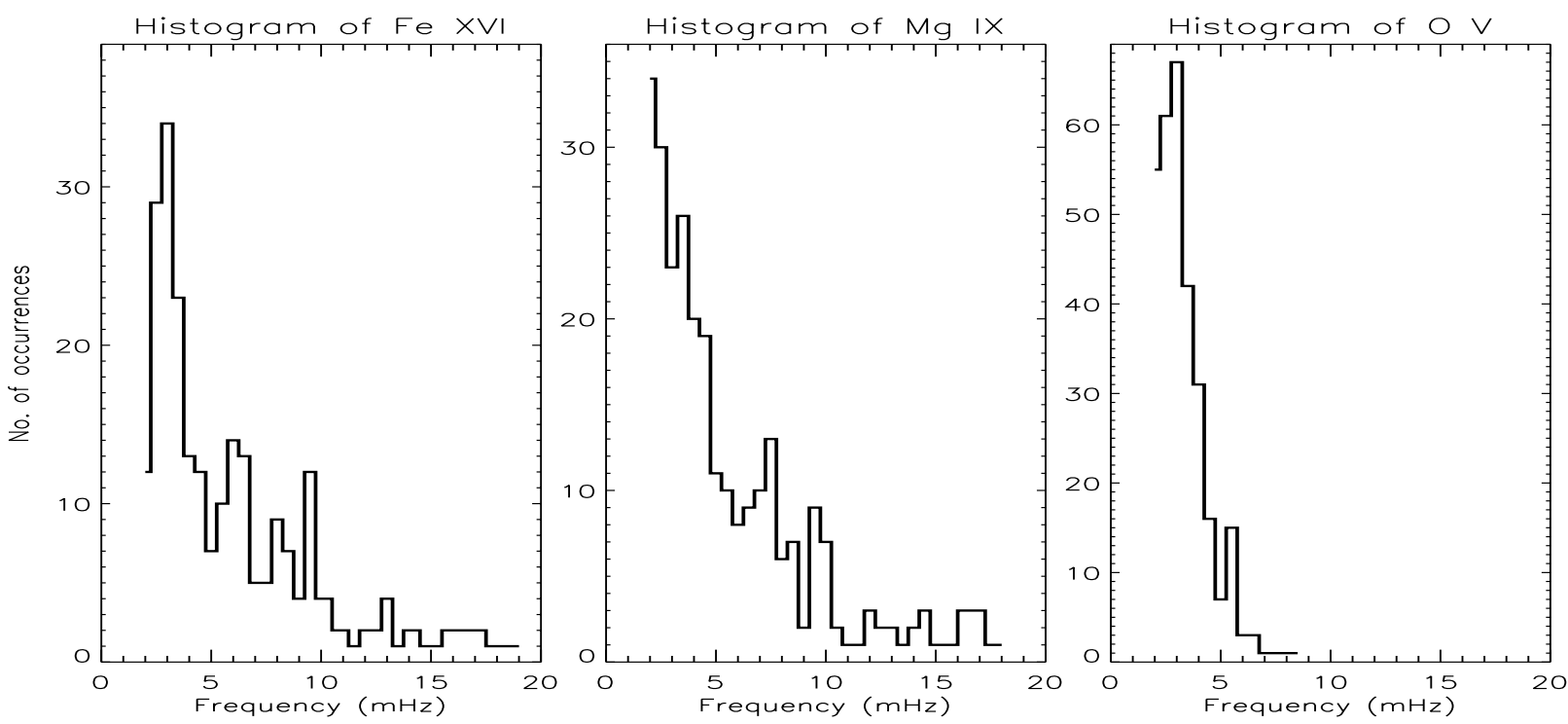

Fig. 9. Histograms of the combined oscillation frequencies, from the primary and secondary oscillations, obtained from the intensity time series of Fexvi $335 \AA$ (left panel), Mg IX $368 \AA$ (middle panel) and Ov $629 \AA$ (right panel)

that causes most of the oscillations to occur with periods around five minutes, i.e. between $2.0-5.0 \mathrm{mHz}(200-500 \mathrm{~s})$.

By combining and plotting the primary and secondary oscillation frequencies in one histogram, as we did with the intensity oscillations, we are able to produce the plot shown in Fig. 11. For Fe XVI we can see that the majority of oscillations are present at the lower frequencies, concentrated at 2.5 and $4.0 \mathrm{mHz}$, with a decreasing number of higher frequencies present all the way up to $20.0 \mathrm{mHz}$. The localised peaks at $2.5,4.0$ and $6.5 \mathrm{mHz}$ etc. possibly indicates that these are favoured frequencies of oscillation for this line.

For the Mg IX line it can be seen from the plot that the majority of oscillations are also present at low frequencies, with concentrations at 2.5 and $4.0 \mathrm{mHz}$. In addition, there are a sizeable number of oscillations present all the way up to $20 \mathrm{mHz}$, but concentrated mainly between 6.0 $15.0 \mathrm{mHz}$. In the $\mathrm{OV}$ line the majority of oscillation are present at 2.5 and $6.0 \mathrm{mHz}$. There are, however, also oscillations present at higher frequencies all the way up to $16.5 \mathrm{mHz}$.

There is a noticeable increase in the number of frequencies present in the velocity histograms of the Mg IX and Fe XVI lines in Fig. 11 in comparison with the intensity histograms in Fig. 9. The numbers in the O v histograms however remain more or less the same. We suggest that this may be due to a change in the nature of the wave mode as we pass from the transition region line of $\mathrm{O} v$ to the coronal lines of Mg IX and Fe XVI. 

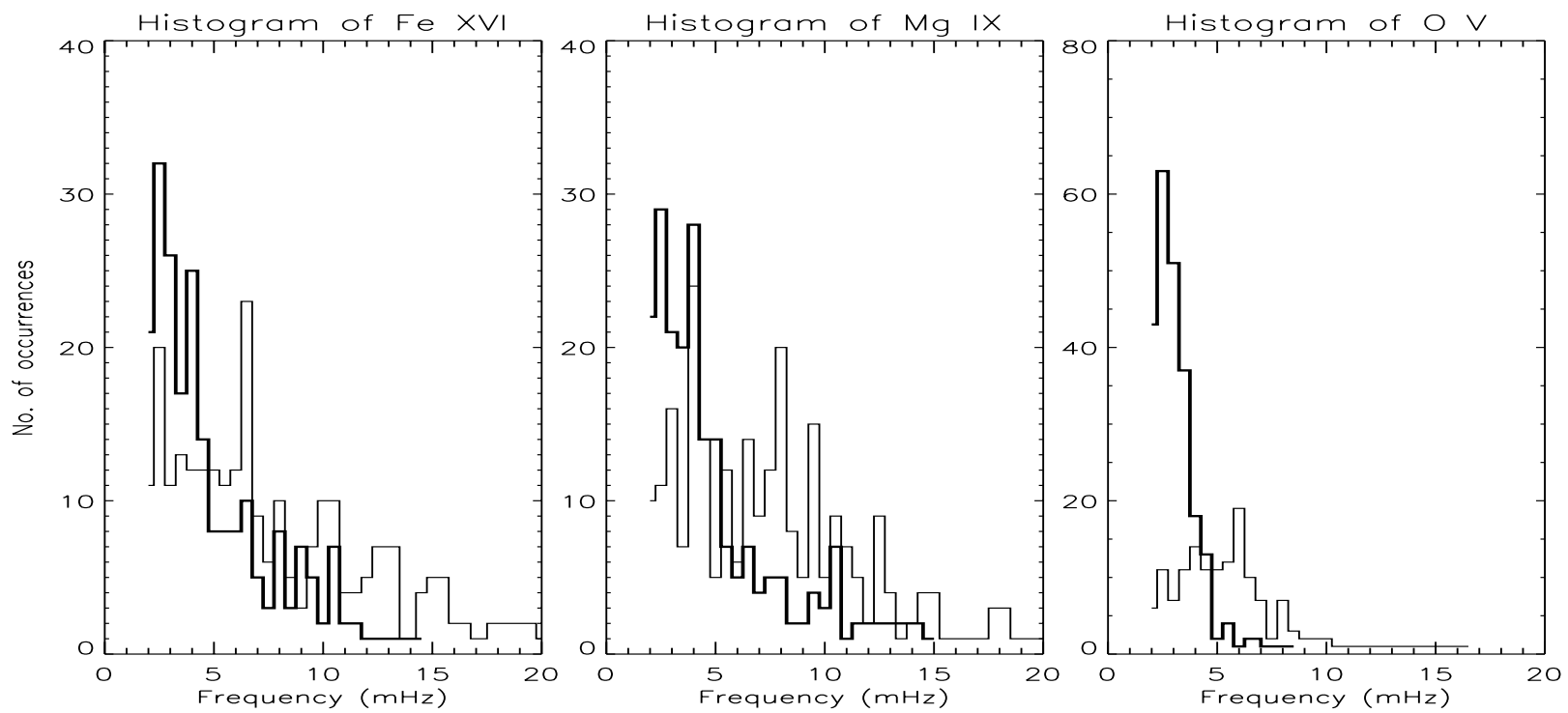

Fig. 10. Histograms of primary oscillation frequencies (bold line) and secondary oscillation frequencies (thin line), obtained from the velocity time series of Fe XVI $335 \AA$ (left panel), Mg IX $368 \AA$ (middle panel) and Ov $629 \AA$ (right panel)
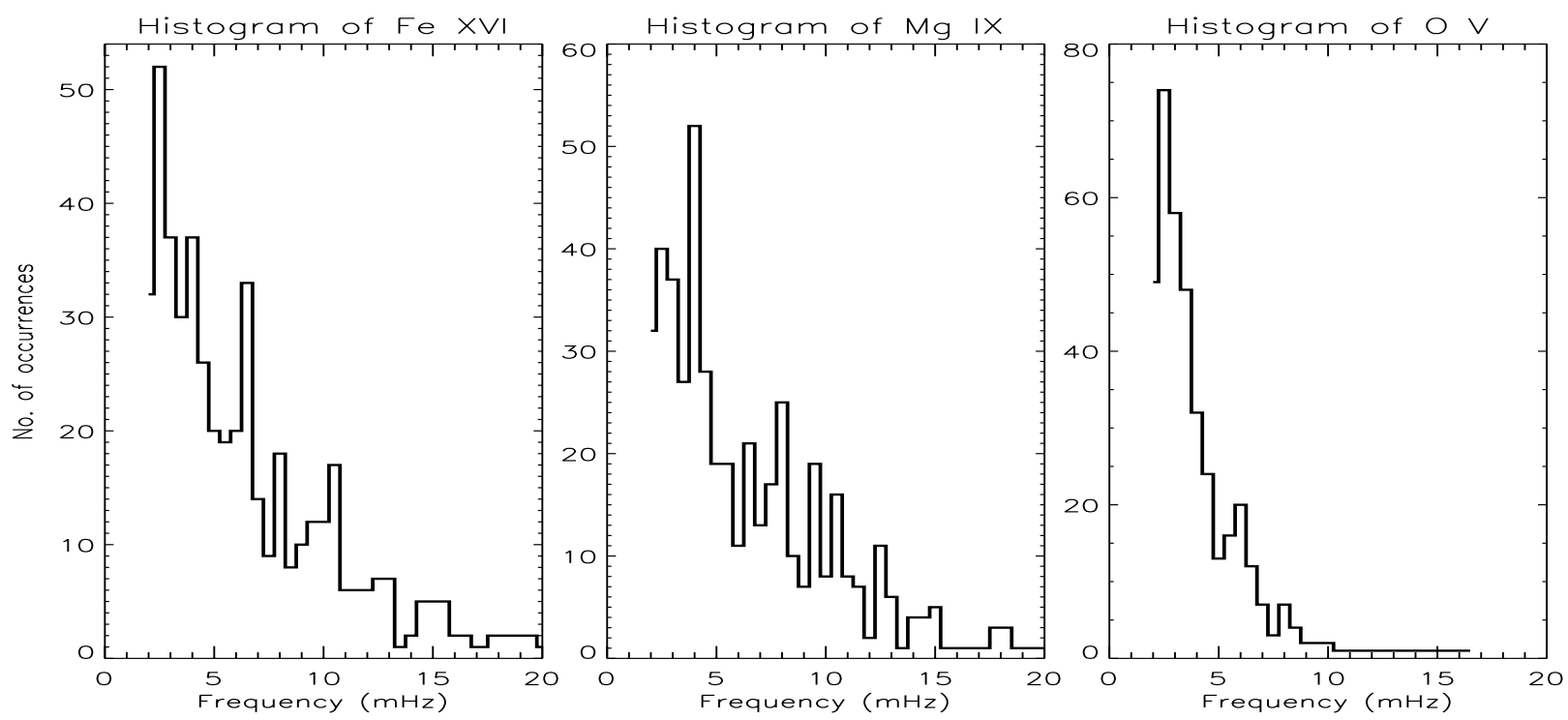

Fig. 11. Histograms of the combined oscillation frequencies, from the primary and secondary oscillations, obtained from the velocity time series of Fe XVI $335 \AA$ (left panel), Mg IX $368 \AA$ (middle panel) and O V $629 \AA$ (right panel)

This and the other results that we obtained here will be discussed further in the next section.

\section{Discussion}

From the detailed analysis of the previous section we found a number of distinct results which we shall discuss in more detail here.

Firstly, we have found from these results that there is a tendency for the higher frequency oscillations, in both the intensity and velocity oscillations, to occur in locations where the total intensity is least. This suggests that the higher frequency oscillations are occurring in the darker regions of the active regions, possibly in the interloop/loop boundary regions and further that some mechanism is act- ing on the loop boundaries. We tentatively note that resonant absorption is likely to occur where inhomogeneities are most pronounced, such as the boundaries of isolated flux tubes.

Secondly, we have seen, by combining the primary and secondary oscillations of intensity and velocity (e.g. Figs. 9 and 11), that the majority of the oscillations in all lines are present in the range of $2-5 \mathrm{mHz}(200-500 \mathrm{~s})$. In fact, typically the largest number of oscillations are found around periods of $\sim 5 \mathrm{~min}$, i.e. $2.5-3.0 \mathrm{mHz}$. The presence of such a large number of low frequency oscillations, with periods around $5 \mathrm{~min}$, in the transition region and the corona strongly suggests the influence, in some manner, of the photospheric five minute oscillations on the upper solar atmosphere. We note that a coupling between the 
photospheric driver and the coronal loop mode would explain the prevalence of the $\sim 5$ min or $\sim 3 \mathrm{mHz}$ peak in all the frequency histograms. Aschwanden et al. (1999) found that a fraction (up to 15\%) of the loops are in resonance with the 5 min driver.

Thirdly, while all lines were found to have a majority of their frequencies in the $2.0-5.0 \mathrm{mHz}$ range, it was noticeable that the MgIX and FexVI lines shared many common characteristics at higher frequencies $(>5.0 \mathrm{mHz})$ in both the intensity and velocity. For example, from the combined histograms of the intensity and velocity oscillations (see Figs. 9 and 11), both Fe XVI and Mg IX can be seen to have most of their higher frequency $(>5.0 \mathrm{mHz})$ oscillations concentrated roughly in the $5.0-10.0 \mathrm{mHz}$ range, with a continuous range of frequencies present all the way out to $\sim 20 \mathrm{mHz}$. Local maxima in the frequency histograms, however, suggest that some higher frequencies may be more favoured than others, e.g. at $6.5 \mathrm{mHz}$ in the Fe XVI velocity histogram. The similarity between the intensity and velocity frequencies of the $\mathrm{OV}$ is also clear from the combined histograms in Figs. 9 and 11. For example, both have most of their oscillations at $2.5-3.0 \mathrm{mHz}$, with a smaller amount weaker at $5.5-6.0 \mathrm{mHz}$. The only slight difference being that in the velocity $\mathrm{OV}$ has more oscillations at higher frequencies, up to $16.5 \mathrm{mHz}$. While overall we find roughly the same frequencies in the intensity and the velocity oscillations it should be noted that the same values of frequency rarely occur simultaneously in both the intensity and velocity measurements of a single region. This was discussed briefly in Sect. 3.1 where we suggested it might be due to the influence of two different wave modes (compressive and non-compressive), each being separately responsible for either the intensity or velocity oscillations.

Fourthly and finally, it is noticeable that Fe XVI and MgIX show more significant oscillations in the velocity than in the intensity. This can be confirmed by comparing Figs. 9 and 11. This larger number of oscillations in the velocity with respect to the intensity for the coronal lines may be indicative of the fact that in the velocity oscillations we are measuring additional wave modes that are not visible in the intensity. This would suggest that this additional wave mode is of a non-compressive type, such as an Alfvén wave, that would not be easily measured in the intensity oscillations. However the fact that there are some oscillations in the intensity in the coronal lines suggests that there are also compressive wave types present, possibly magnetoacoustic in nature. For the $\mathrm{O} v$ line there is not an appreciable difference in the numbers of oscillations measured in the intensity and the velocity. This can again be confirmed by comparing Figs. 9 and 11. It may be that the waves causing the intensity and velocity oscillations in the $\mathrm{O} v$ line are generally compressive in nature, and therefore are measurable equally in both intensity and velocity measurements. This, of course, then suggests that there is some mechanism at play that is creating additional non-compressive waves in the coronal lines but not in the transition region line of $\mathrm{O} v$. We do not speculate further here on the nature of this proposed mechanism except to point out that resonant absorption in the coronal loops would be expected to produce kinetic Alfvén waves (Ionson 1978).

Before we briefly discuss the above results in terms of possible theoretical mechanisms, we compare our results to similar findings by other authors (see also Table 2).

Koutchmy et al. (1983) found periods of 43, 80 and $300 \mathrm{~s}$ in Doppler velocities measured using the Fe XVI green coronal line at $5303 \AA$. These were discussed by them in terms of resonant Alfvén oscillations of a coronal arch. Using estimated values for the coronal density, magnetic field and loop length they found periods of $84.5 \mathrm{~s}$ and $43 \mathrm{~s}$ for the first and second modes which closely match their observations.

McKenzie \& Mullan (1997) found periods of 10-62 s for coronal loops measured with the YOHKOH soft X-ray telescope. They suggested that these oscillations were due to the loop oscillating in its global mode.

Ruderman et al. (1997) proposed that if a resonant torsional Alfvén wave with a single frequency heats a whole coronal loop, the driving frequency corresponding to this resonance is given by

$\omega=6.8510^{16} B_{\mathrm{o}} n_{\mathrm{e}}^{-1 / 2} L^{-1} \mathrm{~s}^{-1}$.

Using $B_{\mathrm{o}}=5 \mathrm{G}, n_{\mathrm{e}}=10^{9} \mathrm{~cm}^{-3}$ and $L=200000 \mathrm{~km}$ one obtains $\omega=0.0542$ corresponding to a period of $116 \mathrm{~s}$. Decreasing the loop length results in a linear decrease in the calculated period.

Roberts et al. (1984) suggested that the observations found by Koutchmy et al. (1983) could also be interpreted as standing fast kink waves in coronal loops. For the fast kink mode (standing fast magneto-acoustic waves), which would correspond to lateral displacements of the loop, the periods are given by,

$\tau_{\text {kink }}=2 L / j c_{\mathrm{k}}, c_{\mathrm{k}}=\left(\frac{2}{1+\rho_{\mathrm{o}} / \rho_{\mathrm{i}}}\right)^{1 / 2} c_{A}$

where $c_{A}$ is the Alfvén speed inside the loop, $c_{k}$ is the speed of propagation of the kink mode, $\rho_{\mathrm{i}}$ and $\rho_{\mathrm{o}}$ are the plasma densities inside and outside the loop respectively, and $j$ determines the number $(j-1)$ of nodes in the oscillation along the loop, with $j=1$ when the apex of the loop is disturbed whereas for $j=2$ the apex is undisturbed. The modes $j=1,2$ are the most easy to excite. Using the values as reported by Nakariakov et al. (1999) of $c_{\mathrm{A}}=B_{\mathrm{i}} / 4 \pi \rho_{\mathrm{i}}=800 \mathrm{~km} \mathrm{~s}^{-1}$ and assuming an order of magnitude density enhancement for a typical coronal loop, i.e $\rho_{\mathrm{o}} / \rho_{\mathrm{i}}=0.1$, and the length of the loop as $L=130000 \mathrm{~km}$, periods of 240 and $120 \mathrm{~s}$ are obtained for $j=1,2$ respectively. Clearly for a smaller loop length, one will have shorter periods and consequently higher frequency oscillations in the entire loop, e.g. the compact SXR-bright flare loops have lengths $L \sim 20000 \mathrm{~km}$ (Aschwanden et al. 1999).

From TRACE observations, Nakariakov et al. (1999) interpreted their in-phase displacement in a coronal loop as 
Table 2. Summary of previous results

\begin{tabular}{|l|l|l|}
\hline Authors & Lines & $\begin{array}{l}\text { Main Periods } \\
(\mathrm{sec})\end{array}$ \\
\hline Koutchmy et al. (1983) & Fe XVI 5303 A & $43,80,300$ \\
\hline McKenzie and Mullan (1997) & Soft X-rays & $10-62$ \\
\hline Ireland et al. (1999) & $\begin{array}{l}\text { Fe XVI 335 } \\
\text { Mg IX 368 } \\
\text { O v 629 } \AA \\
\text { (CDS) }\end{array}$ & $\begin{array}{l}60-70 \\
\text { no periods } \\
160-200\end{array}$ \\
\hline Aschwanden et al. (1999) & Fe IX (TRACE) & 280 \\
\hline Cowsik et al. (1999) & Continuum & $90,25,7$ \\
\hline
\end{tabular}

being caused by the global kink mode. This is the normal mode of the loop in which all parts of the loop oscillate transversely and in phase. They also observed a decay in the loop oscillations which could be attributed to dissipation of the resonant global mode due to viscosity and resistivity. The waves can be dissipated in a narrow dissipation layer inside the loop (estimated to be $\sim 15 \mathrm{~km}$ ), which leads to heating of coronal loops. The effect of viscous dissipation on the resonant absorption of the global mode has been investigated by Ofman et al. (1994).

Aschwanden et al. (1999) found periods of approx. $280 \mathrm{~s}$ for spatial oscillations of coronal loops measured using the Fe IX $171 \AA$ line of TRACE. Due to the high spatial resolution of TRACE the oscillations were detectable as transverse (lateral) displacements of spatial loop positions. After investigating various MHD modes of coronal loops, they concluded that the observed periods of the asymmetric, sinusoidal displacements are most consistent with the fast kink mode. They also found little phase shift along the loops, implying a standing wave mode with fixed nodes.

From eclipse observations, Singh et al. (1997) and Cowsik et al. (1999) reported intensity oscillations with periods $\sim 5-100 \mathrm{~s}$. These were interpreted as compressional magneto-acoustic waves. Furthermore, they showed that the fast mode oscillations in active regions can provide sufficient energy flux for heating of the solar corona, as was concluded earlier by Porter et al. (1994).

Ireland et al. (1999), using FeXVI $335 \AA$ and MgIX $368 \AA$ lines from CDS, found periods of $60-70 \mathrm{~s}$ for the Fe XVI line, but no significant periods for the Mg IX line. In addition, they found large numbers of oscillations around 160-200 s for the O v $629 \AA$ line.

Moortel et al. (2000) using the TRACE Fe IX line found propagating variations in the intensity of a coronal loop footpoint. They measured these intensity variations to have periods of $180-240 \mathrm{~s}$. From the propagation speed of these intensity variations $\left(\sim 150 \mathrm{~km} \mathrm{~s}^{-1}\right)$ they consider the oscillations to be good candidates for being propagating slow magneto-acoustic waves travelling into the corona. They further point out that, unlike the observations of Aschwanden et al. (1999), no flare occurred near the time of their observations.

Recently, Nightingale et al. (1999) studied the time variability of EUV brightenings in coronal loops with
TRACE. The brightenings were interpreted as being produced by compressional waves that travel with the sound speed in coronal loops. They found an electron density compression by a factor of $1.3-2.0$ with respect to the ambient plasma, but did not find any signature of heating. These authors concluded that the brightenings were related to some physical process distinctly different from the flare-like process, where a density increase correlates with a temperature increase. Transient EUV brightenings propagating with speeds of $75-200 \mathrm{~km} \mathrm{~s}^{-1}$ along coronal loops have also been reported by Berghmans \& Clette (1999) from EIT observations. It is interesting to note that Pérez \& Doyle (2000) reported transition region electron density enhancements in the range 1.5-2.0 (based on a O IV electron density sensitive line pair), which they suggested to be related to ultraviolet explosive events.

We will now compare our observed values with the values discussed above, some of which are also shown in Table 2. In Sect. 3.2 we saw that oscillations at frequencies between $2-5 \mathrm{mHz}$, that is, period of $200-500 \mathrm{~s}$ are common in all the lines. In addition, we found that the Fe XVI and Mg Ix lines shared roughly the same frequency bands in both the intensity and the velocity. Unfortunately due to the wavelet filtering applied by us, higher frequencies $(>20 \mathrm{mHz}$ ) could not be distinguished from noise, and so the lowest periods that could be found by us are at $\sim 50 \mathrm{~s}$. It is impossible therefore to compare with the lowest periods found in the literature, for example, the $43 \mathrm{~s}$ period found by Koutchmy et al. (1983) or the values of 25 and $7 \mathrm{~s}$ found by Cowsik et al. (1999). However, the few frequencies larger than $10 \mathrm{mHz}$ (<100 s periods), found in the high frequency tails of the MgIX and Fe XVI histograms in Figs. 9 and 11 are close in value to the upper range of periods found by McKenzie \& Mullan (1997), the value of $80 \mathrm{~s}$ found by Koutchmy et al., the value of $90 \mathrm{~s}$ found by Cowsik et al. and the values of $60-70 \mathrm{~s}$ found by Ireland et al. for the Fe XVI line. The period of $280 \mathrm{~s}$ measured by Aschwanden et al. (1999), the $300 \mathrm{~s}$ period measured by Koutchmy et al., the 180-240 s periods found by Moortel et al. (2000) and the 160-200 s period found by Ireland et al., for their $\mathrm{OV}$ line measurements, all easily fall into the range of the ubiquitous low frequency $2-5 \mathrm{mHz}(200-$ $500 \mathrm{~s}$ ) oscillations measured in our analysis.

It is difficult from the results presented here to know which mechanism is responsible for the observed oscillations, and, indeed, the different frequencies found by us may be indicative of more than one wave mode or mechanism being responsible for the observed oscillations. From our results we find that higher frequency oscillations tend to occur preferentially in the interloop/loop boundary regions, possibly as the result of some resonant absorption process. In the high temperature coronal lines, we find more significant oscillations in the velocity than in the intensity, which suggests that in the velocity we measure additional non-compressive wave modes not visible in the intensity. As this effect is not seen in the transition region line of $\mathrm{OV}$ it suggests that these additional noncompressive modes are produced in and limited to the 
corona. In addition, we find that a $\sim 5$ min oscillation signature is commonly present in all lines, strongly suggesting a coupling of the photospheric driver with the transition region and coronal loop modes. We conclude from our results and from the above discussion, that there are two main mechanisms responsible for the observed oscillations; either resonant Alfvén and/or fast kink waves or propagating slow magnetoacoustic waves (Moortel et al. 2000), both present in coronal loops.

Acknowledgements. Research at Armagh Observatory is grantaided by the Dept. of Education for N. Ireland while partial support for software and hardware is provided by the STARLINK project which is funded by the UK PPARC. Information on the wavelet filtering program, MR/1, may be found at www.multiresolution.com. Wavelet software was provided by C. Torrence and G. Compo, and is available at URL: http://paos.colorado.edu/research/wavelets/. This work was supported by PPARC grant GR/K43315 plus a shortterm fellowship to DB from Armagh Observatory. EOS and BF are members of the European Solar Magnetometry Network (www.astro.su.se/ dorch/esmn/). We would like to thank the CDS team at Goddard Space Flight Center for their help in obtaining the present data. CDS is part of SOHO, the Solar and Heliospheric Observatory, which is a mission of international cooperation between ESA and NASA.

\section{References}

Aschwanden, M. J., Fletcher, L., Schrijver, C. J., \& Alexander, D. 1999, ApJ, 520, 880

Berghmans, D., \& Clette, F. 1999, Solar Phys., 186, 207

Cowsik, R., Singh, J., Saxena, A. K., Srinivasan, R., \& Raveendran, A. V. 1999, Solar Phys., 188, 89

Dhillon, V. S., \& Privett, G. J. 1997, Starlink User Note, 167.5
Doyle, J. G., van den Oord, G. H. J., O'Shea, E., \& Banerjee, D. 1998, Solar Phys., 181, 51

Doyle, J. G., van den Oord, G. H. J., O'Shea, E., \& Banerjee, D. 1999, A\&A, 347, 335

Harrison, R. A. 1997, Solar Phys., 175, 467

Hollweg, J. V. 1990, Computer Science Reports, 12, 205

Ionson, J. 1978, ApJ, 226, 650

Ireland, J., Walsh, R. W., Harrison, R. A., \& Priest, E. R. 1999, A\&A, 347, 355

Koutchmy, S., Žugžda, Y. D., \& Ločans, L. 1983, A\&A, 120, 185

Meyers, S. D., Kelley, B. G., \& O’Brien, J. J. 1993, Mon. Wea. Rev., 121, 2858

De Moortel, I., Ireland, J., \& Walsh, R. W. 2000, A\&A, 355, L23

McKenzie, D. E., \& Mullan, D. J. 1997, Solar Phys., 176, 127

Nakariakov, V. M., Ofman, L., DeLuca, E. E., Roberts, B., \& Davila, J. M., Science, 285, 862

Narain, U., \& Ulmschneider, P. 1996, Space Sci. Rev., 75, 453

Nemec, A. F., \& Nemec, J. M. 1985, AJ, 90, 2317

Nightingale, R. W., Aschwanden, M. J., \& Hurlburt, N. E. 1999, Solar Phys, 190, 249

Ofman, L., Davila, J. M., \& Steinolfson, R. S. 1994, ApJ, 421, 360

Pérez, M. E., \& Doyle, J. G. 2000, A\&A, 360, 331

Porter, L. J., \& Klimchuk, J. A., \& Sturrock, P. A. 1994, ApJ, 435,482

Roberts, B., Edwin, P. M., \& Benz, A. O. 1984, ApJ, 279, 857

Ruderman, M. S., Berghmans, D., Goossens, M., \& Poedts, S. 1997, A\&A, 320, 305

Singh, J., Jagdev, C. R., Raveendran, A. V., et al. 1997, Solar Phys., 170, 235

Starck, J. L., \& Murtagh, F. 1998, PASP, 110, 193

Starck, J. L., Murtagh, \& F., Bijaoui 1998, Image and Data Analysis: The Multiscale Approach (Cambridge University Press)

Torrence, C., \& Compo, G. P. 1998, Bull. Amer. Meteor. Soc., 79,61 